\title{
Acné vulgaris: actualizaciones en fisiopatología y tratamiento.
}

\section{Acne vulgaris: \\ updates on pathophysiology and treatment.}

Pág. 52,65

Recibido: $27-05-2020$

Aceptado: 18-06-2020

Dra. Roxana Fernández Vaglio ${ }^{1}$

Dra. Natalia Pérez Céspedes ${ }^{2}$

1. Médico General, Trabajador independiente, San José, Costa Rica.

2. Médico General, Trabajador independiente, San José, Costa Rica.

\section{RESUMEN}

El acné es una patología dérmica relativamente común que afecta la unidad pilosebácea. Principalmente, afecta a la población adolescente y adultos jóvenes, sin embargo, el cuadro clínico puede afectar también a otros grupos etarios. La etiología del acné es multifactorial y, se han identificado cuatro mecanismos involucrados en el desarrollo de la enfermedad. A pesar de que hoy en día se han estudiado y documentado avances importantes en materia del tratamiento de esta patología, uno de los pilares continúa siendo los retinoides, al que se le pueden adicionar otros medicamentos como el peróxido de benzoilo y los antibióticos.

\section{PALABRAS CLAVES}

Acné vulgar, glándula sebácea, Propionibacterium acnes, retinoides

\section{ABSTRACT}

Acne is a dermatologic pathology relatively common that affects the pilosebaceous unit. It mainly affects adolescents and young adults; however, symptoms may also affect other age groups. The etiology of acne is multifactorial, and four mechanisms involved in the development of the disease have been identified. Despite the fact that advances in terms of treatment have been studied and documented, retinoids remain as one of the main drugs in the treatment of the disease. Drugs such as benzoyl peroxide and antibiotics can be added to the treatment.

\section{KEYWORDS}

Acne vulgaris, sebaceous gland, Propionibacterium acnes, retinoids 


\section{INTRODUCCIÓN}

La piel es el mayor órgano del cuerpo, cumple funciones de protección, ya sea ante diversos patógenos, evitando pérdida de agua o contra múltiples mecanismos de trauma. De igual manera posee gran cantidad de terminaciones nerviosas que proveen sensibilidad; tiene un rol en termorregulación y sintetiza vitamina $D(1,2)$. También es importante recalcar que la piel se encuentra colonizada por una compleja y variada microbiota (3). Anatómicamente se estudia la piel en dos compartimentos: epidermis y dermis. Es la capa profunda de la dermis la que contiene una serie de estructuras especializadas entre las que se encuentran los folículos pilosos, las glándulas sebáceas y sudoríparas $(1,3)$.

El acné (proviene del griego "acmé" que significa punto o mancha), también conocido como acné vulgaris, es una enfermedad crónica inflamatoria que involucra la unidad pilosebácea $(4,5)$. Con una etiología multifactorial, afecta aproximadamente a un $85 \%$ de la población cuya edad oscila entre los 12 y 25 años. Esta prevalencia transcurre sin importar el estatus socioeconómico, nacionalidad o sexo (6); sin embargo, el curso de esta patología es más severa en hombres (7). Existen varios tipos de acné además del vulgaris como lo son acné rosáceo, acné cosmético, acné fulminans y acné mecánica (5).

Típicamente, la clínica de este cuadro se caracteriza por comedones, pápulas y pústulas; su presentación, así como la presencia de otros síntomas puede variar según la clasificación de este (8). El diagnóstico de acné conlleva no solamente secuelas a nivel dermatológico como cicatrices o hiperpigmentación, sino que se asocia a un impacto negativo que afecta directamente la salud mental de los pacientes, alterando su autopercepción e incidiendo inclusive financieramente en este grupo poblacional (8).

El objetivo de esta revisión bibliográfica es orientar al personal de salud en el entendimiento de la fisiopatología, diagnóstico y tratamiento de una patología de alta incidencia poblacional y que esto permita manejar al paciente de manera oportuna con herramientas actualizadas y favorecer así un abordaje biopsicosocial adecuado.

\section{MATERIALES Y MÉTODOS}

El presente trabajo de investigación consiste en una revisión bibliográfica basada en el análisis de literatura científica actualizada, obtenida a través de las siguientes bases de datos: Research Gate (https://www.researchgate. net), The New England Journal of Medicine (https://www.nejm.org), Pubmed (https://www. ncbi.nlm.nih.gov/pubmed), Springerlink (https:// link.springer.com), ScienceDirect (https://www. sciencedirect.com), Google Académico (https:// scholar.google.es/schhp?hl=es), Wiley (https:// onlinelibrary.wiley.com/). La búsqueda se realizó utilizando los términos citados a continuación: "acné vulgaris", "impacto psicosocial acné", "fisiología sistema tegumentario", "fisiopatología acné vulgaris", "tratamiento acné", "retinoides". Así mismo, se utilizaron las palabras "and", "or", "y", "o" como conectores de la búsqueda. La escogencia de la literatura utilizada se sometió a criterios de exclusión para asegurar la calidad del trabajo. Se aceptaron escritos originales, guías de manejo, revisiones bibliográficas y sistémicas; publicados tanto en idioma inglés como en español. Solamente se consideraron aquellos cuya fecha de publicación data de los últimos cinco años a partir del presente. 


\section{GENERALIDADES ACNÉ VULGARIS}

\section{PATOGÉNESIS}

Se reconocen cuatro mecanismos claves involucrados en el desarrollo del acné: (a) aumento en la producción de sebo por agrandamiento de glándula sebácea por efecto androgénico, (b) hiperqueratinización del infundíbulo folicular secundario a aumento de andrógenos, (c) colonización y proliferación de la bacteria Cutibacterium acnes (previamente conocida como Propionibacterium acnes) en el ducto y (d) respuesta inflamatoria asociada tanto a inmunidad innata como adquirida $(4,9)$.

La patogénesis del acné es multifactorial y diversosfactores exacerbantesse han asociadoy estudiado a esta condición: dieta, menstruación, sudor, estrés, ambientes húmedos, edad, uso de productos de higiene diaria, medicamentos tópicos u orales (litio, fenitoína, andrógenos, glucocorticoides), incluso ocupación; entre otros $(7,10)$. No existe una clara relación entre el fumado de tabaco y el acné, existen estudios observacionales que se contradicen entre el aumento de la prevalencia y asociación negativa (11).

Una dieta con alta carga glicémica, aumenta la secreción de andrógenos debido a niveles altos de insulina. El aumento de andrógenos en sangre se asocia a producción de sebo, crecimiento de la glándula sebácea e hiperqueratinzación. Este mismo efecto sobre los andrógenos lo desencadena niveles elevados en plasma del factor de crecimiento insulínico tipo 1 (IGF-1). El IGF-1 se eleva por el consumo de leche y depende no tanto de su contenido de grasa sino por la presencia de hormonas y otras moléculas bioactivas (12).
No solamente alimentos con alta carga glicémica como se han asociado a la patogénesis de acné. Se ha estudiado la influencia de las vitaminas $A, B$ y $D$ en el acné, también se ha observado el efecto de los ácidos grasos, polifenoles, y el zinc. Sus efectos se mencionaran más adelante (13).

Los pacientes con acné suelen tener antecedentes heredofamiliares positivos por esta patología dermatológica (11). Sin embargo, se conoce poco sobre los mecanismos hereditarios de esta predisposición, se han asociado de momento genes como cyt-P450$1 \mathrm{~A} 1$ y esteroide-21 hidroxilasa, cuyo quehacer se relaciona con la producción de andrógenos (7).

El acné también puede tener causas endocrinológicas, quienes, a su vez, constituyen diagnósticos diferenciales, debido a la similitud de sus manifestaciones dermatológicas. Entre ellas se encuentran el síndrome de ovario poliquístico (SOPQ), la hiperplasia adrenal congénita (HAC), el síndrome seborrea-acnéhirsutismo-alopecia androgenética (SAHA) y síndrome hiperandrogenismo-resistencia a la insulina-acantosis nigricans (HAIR-AN) (14). En el caso de las mujeres, también debe contemplarse como causa el uso de contracepción hormonal oral (9).

\section{EPIDEMIOLOGÍA}

El acné vulgaris tiene una prevalencia en la población, que oscila entre los 12 y 25 años, de aproximadamente $85 \%$ e incluso más; esto lo convierte en una de las tres patologías dermatológicas más frecuentes, según se ha documentado en estudios realizados en Estados Unidos, Francia y Reino Unido $(6,7)$. De este porcentaje, solamente un $40 \%$ buscará 
asesoría médica, el porcentaje restante cursará con un cuadro leve (7). Cerca de un $20 \%$ de la población afectada desarrollo acné severo ocasionando cicatrices como secuelas (5).

También, puede presentarse acné neonatal en un $20 \%$, principalmente durante las primeras cuatro semanas de vida; sin embargo, suele resolver de manera espontánea (7). De igual manera, esta patología puede persistir hasta la adultez, un $26 \%$ de mujeres y un $12 \%$ de hombres han reportado persistir con acné en sus cuarenta años (4). Su incidencia es inferior en afroamericanos y asiáticos que en caucásicos (5).

\section{FISIOPATOLOGÍA DEL ACNÉ VULGARIS}

El acné es una patología que involucra la unidad pilosebácea, la cual está conformada por pelo, folículo piloso y la glándula sebácea $(4,15)$. La glándula sebácea se encarga de la producción del $90 \%$ de los lípidos de la superficie de la piel (5); su distribución es predominante en cara y torso, mismas regiones donde con frecuencia se presentan las lesiones por acné (7).

A nivel molecular, se ha visto que en personas con acné, se da una sobreexpresión del complejo mTORC1. Este complejo, que también se ha relacionado con enfermedades como la diabetes tipo 2 , obesidad y resistencia a insulina, tiene como función la regulación del crecimiento celular, proliferación y homeostasia metabólica. Una vez activada la vía mTORC1, se da la activación del factor SREBP1 el cual lleva a la lipogénesis sebácea y además la se da un aumento de secreción androgénica(13) .

En la enfermedad también se ha observado que existe una disminución en la expresión del factor FoxO1 el cual se relaciona con la supresión de andrógenos y el antagonismo del factor SREBP1. Se ha visto que los niveles aumentados de insulina y factor de crecimiento insulínico tipo 1(IGF1) llevan a la activación de la vía del fosfoinositol-3 kinasa/AKT que originan la disminución de FoxO1. Como resultado de esto, se ha visto la transactivación de receptores androgénicos, comedogénesis, aumento en la lipogénesis sebácea e inflamación folicular (13). Según lo previamente descrito, el acné se presenta a partir de la sobreactividad de la glándula sebácea que aumenta a su vez la producción de sebo, de la hiperqueratinización folicular, colonización del C. acnes e inflamación (15).

Sobreproducción de sebo. La producción de sebo toma lugar en los lóbulos sebáceos y alcanza la superficie a través del infundíbulo (7). La glándula sebácea es la estructura de la piel que posee mayor cantidad de receptores para andrógenos, siendo la testosterona la hormona con mayor asociación al acné (7). Recientemente, se han estudiado otros receptores como los activados por proliferadores de peroxisomas, receptor del factor de crecimiento similar a insulina-1 y receptor de leptina (16).

Durante la pubertad, hay un aumento considerable de andrógenos y con ello la producción de sebo (7). A lo largo del recorrido que realiza el sebo hacia la superficie, suple de ácido linoleico los queratinocitos del folículo piloso. Lo anterior genera un estado de deficiencia local de ácido linoleico a nivel de la barrera folicular, este deterioro en el epitelio barrera favorece la entrada al folículo por parte de ácidos grasos libres formados por $\mathrm{C}$. acnes (7).

Los ácidos grasos libres estimulan la producción de varias citocinas, generando así una reacción inflamatoria local (7). 
Hiperqueratosis folicular. Una función del sebo es servir de medio de transporte hacia la superficie para los queratinocitos sueltos, ubicados dentro del folículo piloso. De esta manera, existe una descamación regular en equilibrio con la formación constante de queratinocitos. En el acné existe proliferación excesiva de queratinocitos, lo que conlleva a la hiperqueratosis folicular (7).

Colonización de C. acnes. La presencia de C. acnes no es por sí mismo el mecanismo principal del acné. Una serie de cambios en el ambiente local, generados por distintos estilos de vida incluyendo hábitos de higiene o dieta, pueden predisponer las condiciones óptimas para la colonización del folículo piloso por parte de esta bacteria (3). Existen otros microorganismos que también componen la microbiota de la piel y pueden contribuir en la patogénesis del acné: Staphylococcus epiermidus y Malassezia furfur (7).

Estado inflamatorio. La secreción de productos pro inflamatorios por parte de C. acnes genera una respuesta inmune tanto humoral como celular, así como activación del complemento (5). El mismo estado inflamatorio desencadena un aumento en la producción de sebo y en la proliferación de queratinocitos (7).

\section{CLASIFICACIÓN Y GRADACIÓN DEL ACNÉ}

De acuerdo con las guías de la Academia Americana de Dermatología, no es posible recomendar un sistema universal de gradación o clasificación del acné (17). Dado lo anterior, existen diversas clasificaciones y gradaciones de severidad del acné, lo cual implica una falta de consenso. A su vez, muchas de estas categorías son subjetivas (18). Dos de las escalas de gradación más utilizadas son la Escala global de evaluación del acné y la escala propuesta por la FDA de los Estados Unidos (19).

En 2016, se publica las guías europeas para el manejo del acné, en estas se divide el abordaje del paciente de acuerdo con el tipo de acné, siendo dividido en 1) comedónico, 2) papulopustular leve a moderado, 3) papulopustular severo/acné nodular moderado y 4) nodular severo/acné conglobata (20). También, en 2016, se realiza el consenso español para clasificación del acné. En este acuerdan una clasificación similar a la europea (21).

Tomando en cuenta lo anterior, Moradi et al, proponen una clasificación de severidad de acuerdo con las formas clínicas del acné. Es el acné leve aquel comedónico o papulopustular en el que no hay nódulos, quistes o tractos sinusoidales y existen menos de 10 lesiones papulopustulares y menos de 20 comedones. El acné moderado abarca el papulopustular y nodular, pero debe presentar 10 a 40 comedones, 10 a 40 lesiones papulopustulares y menos de 10 nódulos. Por último, el acné severo incluye los tipos nodulocistico y conglobata que presenta de 40 a 100 comedones fusionados, más de 40 lesiones papulopustulares y más de 10 nódulos con tractos sinusoidales y quistes (19).

Otra forma en que puede clasificarse el acné de acuerdo con Plewig et al, es dividirlo en 3 subtipos: comedónico, papulopustuloso y conglobata. Una vez tipificado, este debe ser graduado para lo que proponen cuatro grados para cada subtipo (22).

En cuanto a los subtipos comedónico y papulopustuloso, se debe contar las lesiones características de cada uno $\mathrm{y}$, dependiendo del 
número de estas se procederá a asignar el grado. De esta forma se tiene acné grado I (menos de 10 comedones o lesiones papulopustulares), grado II (10 a 25 lesiones), grado III (26 a 50 lesiones) y grado IV (más de 50 lesiones) (22). Por su parte, el acné conglobata es severo y se puede graduar de la siguiente forma: grado I (aquel que se encuentra en una sola región anatómica cara y cuello, pecho, espalda o glúteos), grado II (existe afectación de más de 2 regiones anatómicas), grado III (presencia de drenaje por sinusoides) y grado IV (presencia de cicatrices atróficas o hipertróficas) (22).

\section{TRATAMIENTO}

A grandes rasgos, existe tratamiento tópico, sistémico y otras terapias (23). A la hora de prescribir el tratamiento, es importante guiarse de acuerdo con la clasificación a la que pertenece la enfermedad de cada paciente. Se ha visto que en la mayoría de los pacientes con acné comedónico o con lesiones inflamatorias, el tratamiento de primera línea consiste en el uso de retinoides tópicos junto con peróxido de benzoilo (18).

De acuerdo con las recomendaciones NICE, el acné puede ser tratado en atención primaria, sin embargo, existen indicaciones de referencia a especialista. Dentro de estas se encuentran: personas en riesgo de formación de cicatrices, pacientes con afectación psicosocial, pacientes con casos de acné fulminante o conglobata, aquellos pacientes moderados a severos que no han tenido respuesta al tratamiento y aquellos pacientes en los que se considere el uso de retinoides orales (24).

Para propósitos del presente artículo, primero se mencionan las recomendaciones terapéuticas de acuerdo con la clasificación del acné y, posteriormente se abordará el mecanismo de acción de los medicamentos más utilizados.

\section{1) Acné leve: comedónico y papulopustular}

La evidencia es limitada para el grupo de pacientes con acné comedónico (20). Para este tipo de acné se pueden utilizar retinoides tópicos, se puede considerar el uso de peróxido de benzoilo o ácido azelaico o se puede utilizar una combinación de estos(17, 20). En el caso de pacientes con acné papulopustular, se recomienda el uso de terapia de combinación, la cual incluye peróxido de benzoilo, retinoide y podría utilizarse tratamiento antibiótico tópico $(17,20)$. En aquellos pacientes con acné leve, podría considerarse iniciar tratamiento con monoterapia (peróxido de benzoilo o retinoide tópico) $y$, en caso de no haber respuesta adicionar un segundo agente (4).

\section{2) Acné moderado: papulopustular y nodular}

Para aquellos pacientes en el espectro moderado, se podría considerar el uso de antibióticos orales junto con terapia tópica combinada $(4,25)$, sin embargo, cabe destacar que no se recomienda el uso de antibióticos como monoterapia en ningún paciente (20). Para aquellos pacientes con cuadros moderados en los que no se documente respuesta, podría considerarse el cambio del antibiótico oral, cambio de los componentes tópicos, terapia hormonal en mujeres o el uso de isotretinoína oral (4).

\section{3) Acné severo: papulopustular severo/ nodular moderado, nodular severo/acné conglobata}

En el tratamiento de estos pacientes se puede considerar varias opciones como tratamiento inicial. Se podría considerar el uso de isotretinoína oral como primera línea o se 
podría utilizar una combinación de tratamiento antibiótico oral junto con isotretinoína oral o junto a tratamiento tópico con peróxido de benzoilo y/o retinoide tópico $(4,20,25)$. En el caso de mujeres, se puede considerar el uso de tratamientos antiandrogénicos/hormonales con antibióticos sistémicos y tratamientos tópicos no antibióticos. La terapia hormonal no debe utilizarse como monoterapia (20).

\section{OTRAS TERAPIAS}

Fuera del espectro de tratamiento farmacológicos tópicos o sistémicos, también existen las terapias tópicas dentro de las cuales cabe mencionar terapias a base de luz y láser, exfoliación química y radiofrecuencia fraccionada con microagujas (26). Estas terapias con láser, luz intensa pulsad y la terapia fotodinámica no deben considerarse como tratamientos de primera línea (18).

Dentro de las terapias a base de luz y láser cabe destacar que no existe evidencia adecuada para recomendar su uso (20), sin embargo, estudios limitados han encontrado evidencia de reducción del número de pápulas, pústulas y comedones en pacientes sometidos a luz pulsada intensa, mientras que otros han encontrado evidencia de disminución de inflamación con láser diodo (26). En lo que sí concuerdan las distintas guías de tratamiento es en la recomendación de no utilizar luz UV artificial en el tratamiento de estos pacientes $(20,23)$. La radiofrecuencia fraccionada con microagujas también ha demostrado mejoría de lesiones inflamatorias y no inflamatorias, sin embargo, los estudios no han sido óptimos (26).

Por su parte, al igual que con la terapia que utiliza luz y láser, existe poca evidencia sobre el beneficio de la exfoliación química (17). Pese a esto, existe cierta evidencia que sugiere que los agentes como ácido glicólico, ácido salicílico, ácido tricloroacético entre otros, podrían brindar mejoría de las lesiones (26).

\section{OPCIONES FARMACOLÓGICAS}

\section{TÓPICOS}

\section{PERÓXIDO DE BENZOILO}

Pese a tener propiedades antimicrobianas, no se han reportado casos de resistencia (27). A parte de sus propiedades antibacterianas (produce radicales libres que degradan proteínas bacterianas), también se ha descrito propiedades comedolíticas. A nivel intracelular en los queratinocitos, aumenta la proporción de glutatión oxidado ha reducido $(23,28)$. El medicamento se debe utilizar de forma separada a los retinoides tópicos (28), sin embargo, presenta actividad sinérgica con el adapaleno (23). Algunos de los efectos adversos incluyen ardor, eritema, resequedad y dermatitis de contacto $(27,28)$.

\section{RETINOIDES TÓPICOS}

Dentro de estos medicamentos se encuentra la tretinoína, isotretinoína, adapaleno y tazaroteno $(27,28)$. Estos controlan la formación de microcomedones, disminuyen la producción de sebo, tienen propiedades antiinflamatorias y normaliza la descamación del epitelio (28). Estos medicamentos actúan sensibilizando los queratinocitos a la apoptosis, al aumentar la expresión de p53 y las caspasas proapoptóticas. A su vez, el aumento de p53 reduce señalización de los receptores de andrógeno (23). Al realizar esto, se da una reducción de la obstrucción del folículo (27). Los efectos en lesiones primarias 
de los retinoides tópicos son dosis dependientes, esto se ha comprobado en diversos estudios en los que se ha visto mayor eficacia a concentraciones más altas de retinoides (29). Algunos de los efectos adversos de estos medicamentos incluyen fotosensibilidad e irritación, resequedad, eritema y descamación $(23,27)$.

\section{ANTIBIÓTICOS TÓPICOS}

Como se mencionó previamente, no se recomienda el tratamiento del acné utilizando antibióticos como monoterapia. Los antibióticos utilizados tienen propiedades antiinflamatorios y antibacterianas (23). Se ha visto que existe un aumento en la resistencia a eritromicina (hasta de un $60 \%$ ), por lo que la recomendación actual es la utilización de clindamicina al $1 \%(23,27,28)$. Se ha visto que el uso de peróxido de benzoilo junto al antibiótico tópico puede disminuir el riesgo de aparición de resistencia (30).

\section{SISTÉMICOS}

\section{ISOTRETINOÍNA}

Este derivado de la vitamina $A$ es el único antiacneico que se ha comprobado tiene efecto sobre los 4 mecanismos de desarrollo del acné $\mathrm{y}$, además puede suprimir a largo plazo la enfermedad (28). Su mecanismo de acción involucra el aumento de la expresión de p53 y FoxO1, llevando a la apoptosis de queratinocitos, lo que lleva a una inhibición de la hiperqueratosis. También se ha visto que al haber una regulación al alta de las proteínas FoxO1 y Fox O3, se da la apoptosis de los sebocitos, llevando a una disminución de la producción de sebo (31). Al existir una disminución de la producción sebácea se da un cambio en el microambiente, lo que favorece una disminución de la colonización de folículos por C. acnes (28).

Dentro de los efectos adversos del medicamento cabe destacar la teratogenicidad, prurito, queilitis, fotosensibilidad, rinitis, epistaxis, cefaleas, alteraciones en pruebas de función hepática, anemia, leucopenia, hipercolesterolemia, depresión y se ha reportado suicidio (no se ha establecido relación causal) $(23,27)$. Se ha visto que en algunos pacientes puede existir un empeoramiento del acné al iniciar tratamiento con isotretinoína, esto se puede minimizar iniciando el tratamiento a dosis bajas (18).

Laduración deltratamiento se debe individualizar, puede variar entre 15 a 32 semanas y se puede observar mejoría pese a la suspensión del tratamiento (23). Las guías de la academia americana de dermatología sugieren continuar tratamiento hasta la desaparición completa del acné (18).

\section{ANTIBIÓTICOS}

Lo antibióticos orales más ampliamente utilizados son la doxiciclina y minociclina (23). Sin embargo, existen otros grupos de antibióticos que pueden ser considerados entre los cuales se encuentran los macrólidos, fluoroquinolonas, tetraciclinas y co-trimoxazol (28).

Se considera las tetraciclinas la primera línea de tratamiento en los pacientes en que se utilice antibióticos orales y, se considera que su efecto antiinflamatorio es más importante que el efecto en reducción de los niveles de C. acnes (23).

En cuanto al uso de antibióticos cabe destacar que su eficacia se debe evaluar a las 6 semanas de tratamiento y en caso de no observar mejoría se debe cambiar el antibiótico (27). El uso de antibióticos para el tratamiento del acné se 
ha relacionado con efectos no intencionales como resistencia de $\mathrm{S}$. aureus, aumento del riesgo de faringitis durante 1 año, después del uso de antibióticos y aumento del riesgo de infecciones de vías respiratorias superiores durante 1 año después del tratamiento por al menos 6 semanas. En cuanto a la duración del tratamiento antibiótico, este se debe limitar a 3-4 meses y al descontinuar seguir tratamiento tópico (30).

\section{TERAPIA HORMONAL}

Esta categoría de tratamiento se puede dividir en dos grandes grupos, los medicamentos antiandrogénicos y los anticonceptivos orales combinados.

Dentro de los tratamientos antiandrogénicos se encuentran la espironolactona, flutamida y el acetato de ciproterona (23). Estos medicamentos antiandrogénicos se unen al receptor de andrógenos y funcionan como antagonistas de testosterona y dihidrotestosterona (23). Se utilizan en mujeres en las que exista contraindicación para el uso de anticonceptivos orales o en las cuales estos no hayan funcionado (27).Por su parte, los anticonceptivos orales son útiles tanto para el tratamiento de lesiones inflamatorias como no inflamatorias, sinembargo, su efecto no podría verse hasta luego de 3 a 6 meses de iniciado el tratamiento $(27,28)$. Los anticonceptivos orales reducen la producción de sebo además aumentan la síntesis de globulina ligadora de hormonas sexuales lo que lleva a la disminución de testosterona libre (28).

Se debe mencionar que los anticonceptivos hormonales pueden contener progestinas y estas pueden tener propiedades androgénicas. Existen diversas generaciones de progestinas y su capacidad androgénica va disminuyendo conforme se avanza en generación (32). El acetato de medroxiprogesterona podría exacerbar el acné (18). La FDA ha aprobado 3 formulaciones para el tratamiento del acné, ellas incluyen una progestina (norgestimato, noretindrona o drospirenona) y etinilestradiol (32). El tratamiento se debe utilizar por lo menos por 1 año (28).

\section{OTROS TRATAMIENTOS SISTÉMICOS}

\section{METFORMINA}

La metformina actúa aumentando el p53, al hacer esto lleva a la inhibición de mTORC1, el cual se encuentra aumentado en las glándulas sebáceas de pacientes con acné (23). En 2017, se realiza un estudio en el que se evalúan distintos estudios, si bien limitados, en el que se logra concluir que la metformina podría ser utilizada en el tratamiento conjunto del acné. Sin embargo, existe limitada evidencia respecto a su uso (33).

\section{CORTICOESTEROIDES}

Este tratamiento se limita a los casos de acné conglobata y fulminans. En estos pacientes se podría utilizar prednisolona o algún antibiótico de potencia similar (23).

\section{ACNÉ Y NUTRICIÓN}

En 2017, se publica una revisión sistemática de estudios que valoran el efecto de la nutrición en el acné. Pese a que no existe suficiente cantidad de estudios y el nivel de evidencia no es alto, se concluye que existe evidencia de que los alimentos con alto índice glicémico y la leche pueden tener influencia sobre las manifestaciones del acné (34). La leche contiene muchos componentes los cuales 
pueden contribuir a su acción acneica. Dentro de los mismos cabe mencionar las hormonas progesterona, androstenediona, DHEA y precursores de dihidrotestosterona. A su vez, en la leche se pueden encontrar microARN (en específico el 21 exosomal y el $148^{a}$ ) los cuales promueven la expresión mTORC. Otros de los componentes presentes en la lache promueven la síntesis de IGF-1 y además tienen alto índice insulinémico (13).

Se ha visto que en pacientes con resistencia a la insulina el uso de metformina junto con el consumo de alimentos de bajo índice glicémico puede disminuir el acné. Esta disminución es mayor con el uso conjunto de ambas medidas y se ha visto que es menor si se utilizan las medidas por separado(4).

Existen otros nutrientes o alimentos que se han relacionado con el acné. Las vitaminas se han vinculado con el acné. Las vitaminas del complejo $B$ se adicionan a muchos alimentos (entre ellos la leche) y se ha visto que pueden relacionarse con la aparición de acné. Al contrario del complejo $B$, se ha visto que la vitamina A lleva una disminución de células Th17 y de interleucina 17 lo que puede ser benéfico en el tratamiento del acné. La vitamina $D$ también puede ser beneficiosa en pacientes con acné (13).

Otro componente dietético que se ha vinculado con el acné son los ácidos grasos poliinsaturados. Se ha visto que en pacientes con acné moderado-severo la implementación de dietas ricas en omega 3 y ácido linoleico ha llevado a una mejoría de las lesiones. A diferencia de estos, el omega 6 puede tener efecto proinflamatorio (13).

Existen algunos coadyuvantes dietéticos en el tratamiento del acné. Las moras, las uvas rojas y el té verde actúan reduciendo la lipogénesis de sebo. Los polifenoles (específicamente el resveratrol) inhibe la señalización mTOR y además cuenta con actividad bactericida. Los probióticos orales han sido reportados como benéficos por su efecto en la reducción de citosinas, por su parte los tópicos se encuentran en investigación sin embargo podrían mejorar la función de barrera de la piel y disminuir el contenido de sebo. En pacientes con deficiencia de zinc la administración de este podría ser benéfica debido a su actividad bacteriostática(13).

\section{CONCLUSIONES}

El entendimiento de la etiología multifactorial del acné es clave para brindar un abordaje integral al paciente, el cual involucre educación conductual y asesoría individual para la elección del tratamiento.

Es de suma importancia llegar a un consenso respecto a la clasificación y gradación de la enfermedad. Actualmente existe subjetividad en el proceso de diagnóstico, lo que puede llevar a un tratamiento inadecuado de la enfermedad. Si bien es cierto, existen múltiples tratamientos que se han estudiado para el abordaje del paciente con acné, los retinoides continúan siendo parte fundamental del tratamiento. La forma farmacéutica a utilizar va a depender en gran medida de la clasificación y severidad de la enfermedad del paciente. El campo de la terapéutica, en especial el uso de luz, láser, radiofrecuencia y exfoliaciones, requiere de nuevos estudios que permitan dilucidar de forma más adecuada su eficacia y permita realizar recomendaciones apropiadas respecto a su uso. Los efectos de la nutrición no están del todo dilucidados por lo que este campo se podría beneficiar de nuevos estudios. 
1. Moore KL, Dalley AF, Agur AMR. Anatomía con orientación clínica. 8va edición. Barcelona: Wolters Kluwer; 2017.

2. Abdo JM, Nikolai AS, Milner SM. The applied anatomy of human skin: A model for regeneration. Wound Medicine [Internet] 2020 [consultado el 17 Mayo 2020];28: 1-10. Disponible en: https:// www.sciencedirect.com/science/article/pii/S2213909520300033?via\%3Dihub.

3. Egert M, Simmering R, Riedel CU. The Association of the Skin Microbiota With Health, Immunity, and Disease. CPT Journal [Internet] 2017 [consultado el 17 Mayo 2020]; 102 (1): 6269. Disponible en: https://pubmed.ncbi.nlm.nih.gov/28380682/

4. Zaenglein AL. Acne Vulgaris. N Engl J Med [Internet] 2018 [consultado el 24 Mayo 2020]; 379 (14): 1343-1352. Disponible en: https://www-nejm-org.binasss.idm.oclc.org/doi/full/10.1056/ NEJMcp1702493

5. Mohiuddin AK. A comprehensive Review of Acne Vulgaris. J Clin Pharm [Internet] 2019 [consultado el 18 Mayo 2020]; 1 (1): 17-45. Disponible en: https://innovationinfo.org/articles/ JCP/JCP-103.pdf

6. Lynn DD, Umari T, Dunnick CA, Dellavalle RP. The epidemiology of acne vulgaris in late adolescence. Adolesc Health Med Ther [Internet] 2016 [consultado el 19 mayo 2020]; 7 (1): 1325. Disponible en: https://www.researchgate.net/publication/291392555_The_epidemiology_ of_acne_vulgaris_in_late_adolescence

7. Prasad SB. Acne vulgaris: A review on pathophysiology and treatment. Asian J Pharm Clin Res [Internet] 2016 [consultado el 20 Mayo 2020]; 9 (4): 54-59. Disponible en: https://www. researchgate.net/publication/305542890_Acne_vulgaris_A_review_on_pathophysiology_ and_treatment

8. Heng AHS, Chew FT. Systematic review of the epidemiology of acne vulgaris. Sci Rep [Internet] 2020 [consultado el 18 Mayo 2020] 10 (1): 5754. Disponible en: https://doi.org/10.1038/s41598020-62715-3

9. Tan AU, Schlosser BJ, Paller AS. A review of diagnosis and treatment of acne in adult female patients. International Journal of Women's Dermatology [Internet] 2018 [consultado el 22 Mayo 2020]; 4: 56-71. Disponible en: https://www.ncbi.nlm.nih.gov/pmc/articles/PMC5986265/

10. Ogé LK, Broussard A, Marshall MD. Acne Vulgaris: Diagnosis and Treatment. Am Fam Physician [Internet] 2019 [consultado el 20 Mayo 2020]; 100 (8): 475-484. Disponible en: https://www. aafp.org/afp/2019/1015/p475.html

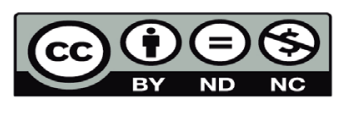


11. Wolkenstein P, Machovcová A, Szepietowski JC, Tennstedt D, Veraldi S, Delarue A. Acne prevalence and associations with lifestyle: a cross-sectional online survey of adolescents/ young adults in 7 European countries. JEADV [Internet] 2018 [consultado el 21 Mayo 2020]; 32: 298-306. Disponible en: https://pubmed.ncbi.nlm.nih.gov/28707712/

12 Ulvestad M, Bjertness E, Dalgard F, Halvorsen JA. Acne and dairy products in adolescence: results from a Norwegian longitudinal study. JEADV [Internet] 2017 [consultado el 21 Mayo 2020]; 31: 530-535. Disponible en: https://www.researchgate.net/publication/305382084 Acne_and_dairy_products_in_adolescence_Results_from_a_Norwegian_longitudinal_study

13. Arias-Gómez MI, Chías-Rubí AE, Adame-Miranda GJ. La importancia de la dieta en el acné. Dermatol Rev Mex [Internet] 2019 [consultado el 10 Jun 2020]; 63(supl.1):S3-S17.

14. Monestel MA. Acné de causas endocrinológicas. Medicina Legal de Costa Rica [Internet] 2016 [consultado el 21 mayo 2020]; 33(1). Disponible en: https://www.scielo.sa.cr/scielo. php?script=sci_arttext\&pid=S1409-00152016000100211\&lng=en\&nrm=iso

15. Kanwar IL, Haider T, Kumari A, Dubey S, Jain P, Soni V. Models for acne: A comprehensive study. Drug Discov Ther [Internet] 2018 [consultado el 18 Mayo 2020]; 12(6):329-340. Disponible en: https://www.researchgate.net/publication/330568653_Models_for_acne_A_comprehensive_ study

16. Dréno, B. What is new in the pathophysiology of acné, an overview. JEADV [Internet] 2017 [consultado el 18 Mayo 2020]; 31 (5): 8-12. Disponible en: https://pubmed.ncbi.nlm.nih. gov/28805938/

17. Zaenglein A, Pathy A, Schlosser B, Alikhan A, Baldwin H, Berson D et al. Guidelines of care for the management of acne vulgaris [Internet]. 2020 [consultado el 17 May 2020]. Disponible en: https://www.jaad.org/article/S0190-9622(15)02614-6/fulltext

18. Thiboutot $D$, Dréno $B$, Abanmi A, Alexis A, Araviiskaia $E$, Barona Cabal $M$ et al. Practical management of acne for clinicians: An international consensus from the Global Alliance to Improve Outcomes in Acne. Journal of the American Academy of Dermatology [Internet]. 2018 [consultado el 18 Mayo 2020];78(2): S1-S23.e1. Disponible en: https://www.jaad.org/article/ S0190-9622(17)32603-8/fulltext

19. Tuchayi S, Makrantonaki E, Ganceviciene R, Dessinioti C, Feldman S, Zouboulis C. Acne vulgaris. Nature Reviews Disease Primers [Internet]. 2015 [consultado el 17 Mayo 2020];1(1). Disponible en: https://pubmed.ncbi.nlm.nih.gov/27189872/

20. Nast A, Dréno B, Bettoli V, Bukvic Mokos Z, Degitz K, Dressler C et al. European evidencebased (S3) guideline for the treatment of acne - update 2016 - short version. Journal of the European Academy of Dermatology and Venereology [Internet]. 2016 [consultado el 16 Mayo 2020];30(8):1261-1268. Disponible en: https://pubmed.ncbi.nlm.nih.gov/27514932/

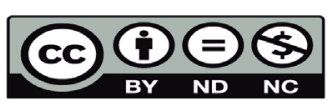


22. López-Estebaranz J, Herranz-Pinto P, Dréno B. Consenso español para establecer una clasificación y un algoritmo de tratamiento del acné [Internet]. 2016 [consultado el 17 Mayo 2020]. Disponible en: https://www.sciencedirect.com/science/article/abs/pii/ S0001731016303313?via\%3Dihu

23. Plewig G, Chen W, Melnik B. Acne Classification and Disease Burden. En: Plewig G, Chen W, Melnick B, ed. por. Plewig and Kligman's Acne and Rosacea [Internet]. 4ta edición. Springer Cham; 2019 [consultado el 15 de Mayo 2020]. p.217-222 Disponible en: https://link.springer. com/chapter/10.1007\%2F978-3-319-49274-2_6

24. Plewig G, Chen W, Melnik B. Acne Therapy. En: Plewig G, Chen W, Melnick B, ed. por. Plewig and Kligman'sAcneandRosacea[Internet].4thed.SpringerCham;2019[consultadoel15Mayo 2020]. p. 223-292. Disponible en: https://link.springer.com/chapter/10.1007/978-3-319-49274-2_7

25. Acne vulgaris - NICE CKS [Internet]. Cks.nice.org.uk. 2020 [cited 11 June 2020]. Available from: https://cks.nice.org.uk/acne-vulgaris\#!scenarioRecommendation

26. Hauk L. Acne Vulgaris: Treatment Guidelines From the AAD. Am Fam Physician [Internet]. 2017 [consultado el 16 Mayo 2020];95(11):740-741. Disponible en : https://pubmed.ncbi.nlm. nih.gov/28671431/

27. de Vries F, Meulendijks A, Driessen R, van Dooren A, Tjin E, van de Kerkhof P. The efficacy and safety of non-pharmacological therapies for the treatment of acne vulgaris: A systematic review and best-evidence synthesis. Journal of the European Academy of Dermatology and Venereology [Internet]. 2018 [consultado el 17 Mayo 2020];32(7):1195-1203. Disponible en: https://pubmed.ncbi.nlm.nih.gov/29444375/

28. Harris V, Cooper A. Modern management of acne. Medical Journal of Australia [Internet]. 2017 [consultado el 18 Mayo 2020];206(1):41-45. Disponible en: https://onlinelibrary.wiley.com/doi/ abs/10.5694/mja16.00516

29. Fox L, Csongradi C, Aucamp M, du Plessis J, Gerber M. Treatment Modalities for Acne. Molecules [Internet]. 2016 [consultado el17 Mayo 2020];21(8):1063. Disponible en: https:// pubmed.ncbi.nlm.nih.gov/27529209/

30. Leyden J, Stein-Gold L, Weiss J. Why Topical Retinoids Are Mainstay of Therapy for Acne. Dermatology and Therapy [Internet]. 2017 [consultado el 17 Mayo 2020];7(3):293-304. Disponible el: https://link.springer.com/article/10.1007/s13555-017-0185-2

31. Adler B, Kornmehl $\mathrm{H}$, Armstrong A. Antibiotic Resistance in Acne Treatment. JAMA Dermatology [Internet]. 2017 [consultado el 16 Mayo 2020];153(8):810. Disponible en: https://pubmed.ncbi. nlm.nih.gov/28636689/

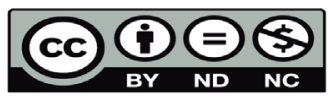


32. Cong T, Hao D, Wen X, Li X, He G, Jiang X. From pathogenesis of acne vulgaris to antiacne agents. Archives of Dermatological Research [Internet]. 2019 [consultado el 17 Mayo 2020];311(5):337-349. Disponible en: https://link.springer.com/article/10.1007\%2Fs00403-01901908-x

33. Bosanac S, Trivedi M, Clark A, Sivamani R, Larsen L. Progestins and Acne Vulgaris: A Review. Dermatol Online J [Internet]. 2018 [consultado el 17 May 2020];24(5). Disponible en: https:// escholarship.org/uc/item/6wm945xf

34. Lee J, Smith A. Metformin as an Adjunct Therapy for the Treatment of Moderate to Severe Acne Vulgaris. Dermatol Online J [Internet]. 2017 [consultado el 18 May 2020];23(11). Disponible en: https://pubmed.ncbi.nlm.nih.gov/29447630/

35. Fiedler F, Stangl G, Fiedler E, Taube K. Acne and Nutrition: A Systematic Review. Acta Dermato Venereologica [Internet]. 2017 [consultado el 18 May 2020];97(1):7-9. Disponible en: https:// pubmed.ncbi.nlm.nih.gov/27136757/ 